\title{
Valores hematológicos y bioquímicos séricos en la raza Perro sin Pelo del Perú
}

\author{
Hematological and serum biochemical values in Peruvian Hairless dogs \\ Giannina Cortés, Ricardo Grandez, Armando Hung
}

\section{RESUMEN}

Objetivo: Determinar los valores del hemograma y bioquímicos séricos para la raza Perro Sin Pelo del Perú (PSPP). Métodos: Se tomó muestras sanguíneas a 91 animales PSPP, luego del análisis en el laboratorio, se obtuvo los valores de hemograma, hematocrito, hemoglobina, y bioquímica sanguínea. También, se recogió información del sexo, tamaño y tipo de alimentación de cada animal. Resultados: La información se resumió mediante estadística descriptiva y las diferencias según sexo, tamaño y tipo de alimentación fueron evaluadas mediante la prueba de $t$ de Student y análisis de varianza. Los resultados muestran que el promedio y rangos de los valores eritrocitarios y leucocitarios estuvieron dentro de rango referencial para la especie; el promedio y rangos de los valores bioquímicos séricos mostraron incremento para los resultados urea y ALT, y resultados por debajo del rango para creatinina y proteínas totales y fraccionadas en comparación con el referencial para la especie; valores elevados de urea y ALT, pero dentro del rango normal, se observó en ejemplares que consumían alimentación mixta. Además Se determinó diferencias significativas para hemoglobina, eritrocitos, segmentados, urea y globulinas en referencia a la variables sexo; asimismo, se encontró diferencias estadísticas para hematocrito, linfocitos, urea, albúmina y globulinas cuando se contrasto con la variable tamaño; y con volumen globular medio (VGM), segmentados, monocitos, urea y proteínas totales cuando se contrastó con la variable alimentación. Los valores encontrados en el presente estudio pueden ser considerados como referencia para la raza PSPP.

Palabras clave: Perro Sin Pelo de Perú, hemograma, bioquímica, renal, hepática

\begin{abstract}
Aim: To determine the complete blood count and serum biochemical values for the Peruvian Hairless Dog (PHD) breed. Methods: A population of 91 PHD canines was obtained, whom they took blood samples to acquire reference values for blood count, hematocrit, hemoglobin, and biochemistry corresponding to the levels of urea and creatinine for renal blood profile, and ALT and GGT for liver profile, total and fractionated proteins for both, being classified by sex, size and type of feeding variables. Results and Conclusions: The Student $T$ test showed significant difference on sex in reference to $\mathrm{Hb}$, erythrocytes, segmented, urea and globulin, and analysis of variance on size reference to Ht, lymphocytes, albumin and globulins, and type of feeding to VGM, segmented, monocytes, urea and total protein, requiring additional controlled studies to determine if they have significance for the breed. It is concluded that the values that we found may be considered as a preliminary references to the PSPP breed.
\end{abstract}

Keywords: biochemical, renal, hepatic, reference values, dogs. 


\section{INTRODUCCIÓN}

Los avances tecnológicos en la especialidad médica veterinaria han ayudado a desarrollar una variedad de análisis de laboratorio complementarias que permiten llegar a un diagnóstico temprano y más específico de muchas enfermedades (Coppo y Mussart, 2000; Sodikoff, 2001). Dentro de estas, las pruebas más comunes son el hemograma y la bioquímica renal y hepática (Coppo y Mussart, 2000). Un estudio retrospectivo reveló que en el 54,5\% de los casos analizados, el diagnóstico inicial fue modificado a partir de los datos obtenidos por pruebas complementarias (Navarro et al., 1999).

El hemograma es parte integral de la investigación para el diagnóstico de cualquier proceso anormal sistémico (Davidson et al., 1998), el cual describe la cantidad y calidad de los elementos celulares en la sangre y algunas sustancias en plasma (Willard et al., 1999; Sodikoff, 2001).

Los componentes bioquímicos de la sangre son muy importantes para la evaluación de la salud animal y su nutrición(Omer, 2009). Las pruebas de funcionamiento renal: nitrógeno ureico sérico, urea y creatinina; y los parámetros bioquímicos referidos a las enzimas que reflejan el daño hepático: alanino aminotransferasa (ALT) y la aspartato aminotransferasa (AST); y las que evalúan colestasis: la fosfatasa alcalina (ALP) y la gamma-glutamil transpeptidasa (GGT) (Giannini et al., 2005); y asimismo la medición de proteínas totales séricas nos ayuda evaluar la función hepática (Willard et al., 1999) permitiendo la valoración de estas funciones.

Los rangos referenciales por especie han sido obtenidos muestreados animales adultos no segregados por edad, sexo o raza (Willard et al., 1999; Ariyibi et al., 2002); observándose modificaciones en estos parámetros por factores como, región geográfica, nutrición o presencia de enfermedades subclínicas (Willard et al., 1999; Ariyibi et al., 2002). Los valores de referencia empleados en la práctica médica recurren a manuales de información veterinaria o publicación de bibliografía extranjera que no siempre se adecuan a las condiciones de regiones con climas específicos (Kaneko et al., 1997; Harvey y Meyer, 2000).

El Perú ha reconocido a el Perro Sin Pelo del Perú (PSPP) como Patrimonio Nacional por el Congreso de la República el 22 de octubre del 2001 y como raza oriunda del Perú mediante Decreto de Ley No. 27537 (Gálvez, 2006). Es la única raza peruana registrada en la Federación Cinológica Internacional (FCI) y fue inscrita en 1985 como raza autóctona del Perú, quedando registrada con el número 310 , dentro del Grupo V, tipo Spitz (Maniero, 2004).

La característica más resaltante de esta raza es la falta total o parcial de pelo, llamado síndrome de hipoplasia ectodérmica (Weiss, 1970); que se transmite como carácter dominante comprendiendo piel, dientes y en algunos casos uñas. Posteriores estudios hacen mención de la presentación de la displasia ectodermal en razas de perros alopécicos, incluyendo al PSPP; asociando la ausencia de pelo debido a la presencia de un gen autosómico dominante, produce descendencia de cachorros normales como alopécicos, que afecta a folículos pilosos, glándulas, uñas y dientes (Mecklenburg, 2006; Kimura et al., 1993).

Teniendo en consideración que las nuevas tendencias en la práctica de la medicina veterinaria se orientan a la prevención y atención de acuerdo a las características de la raza; y asimismo, al aumento de la crianza de la raza PSPP, en nuestro país sino también y a nivel mundial, se hace necesario contar con estudios de la patología clínica en esta raza, siendo el objetivo del presente estudio determinar los valores del hemograma bioquímicos séricos para esta raza.

\section{MATERIALES Y MÉTODOS}

El estudio se realizó en la Facultad de Medicina Veterinaria y Zootecnia (FAVEZ) de la Universidad Peruana Cayetano Heredia (UPCH), Lima, Perú, y recibió aprobación del Comité Institucional de Ética (CIE) de la UPCH. El mismo fue desarrollado entre los meses de mayo a setiembre de 2008 .

El tamaño de muestra considerado fue de un mínimo de 30 perros machos y 30 perros hembras siguiendo el criterio del Teorema del Límite Central a fin de asegurar la normalidad de la distribución de las variables investigadas (Steel et al., 1992). La edad de los animales involucrados en el estudio fue de 2 a 7 años y de condición fisiológica aparentemente sana. Ningún ejemplar hembra estudiado se encontraba en estado de preñez y los machos se eran enteros.

Las fichas de recolección de información contenía la identificación del animal, resultados del examen general clínico (frecuencia respiratoria y cardiaca, y temperatura corporal) y físico (exploración sistemática). Aquellas fichas que presentaban los datos completos y se encontraron dentro de los parámetros normales para la especie canina fueron utilizadas para 
el estudio. Obteniendo así el número total de muestras válidas.

A partir de las fichas consideradas con resultado estándares para la especie respecto al examen clínico y físico general, se recabó los resultados de los análisis sanguíneos del hemograma, hematocrito, hemoglobina, y bioquímica sanguínea correspondiente a los niveles de úrea y creatinina para el perfil renal, y ALT y GGT para el perfil hepático, así como también proteínas totales y fraccionadas para ambos.

Se recolectó muestras de sangre de la vena cefálica con aguja estéril recolectando $3-5 \mathrm{ml}$ de sangre dividido en dos tubos, uno con anticoagulante EDTA y el otro sin anticoagulante para los análisis de hematología y bioquímica, respectivamente. El tubo con EDTA se procesó para las lecturas de hematocrito por medio del método del capilar, hemoglobina con la solución de Drabkin y realizando la lectura en el fotómetro semi-automatizado (Microlab 300), y el recuento de glóbulos rojos y blancos utilizando la solución de Turk y Hayer, respectivamente; así como también la diferenciación leucocitarias con un frotis laminal y tinción Wright.

Del tubo sin anticoagulante se obtuvo suero al centrifugar la muestra a $2500 \mathrm{rpm}$ por 5 minutos. El cual se procesó para obtener los niveles de úrea, creatinina, ALT, GGT, proteínas totales y fraccionadas determinadas por el método colorímetro usando el fotómetro (Microlab 300) con kits comerciales (HUMAN y ELITECH).

Las variables fueron divididas en grupos: sexo, macho y hembra; tamaño, pequeño ( $>3 \mathrm{y} \leq 8 \mathrm{~kg}$.), mediano ( $>8 \mathrm{y} \leq 15 \mathrm{~kg}$.) y grande $(>15 \mathrm{y} \leq 30 \mathrm{~kg}$.); y tipo de alimentación, comida casera, mixta (casera y balanceada) y balanceada (alimento balanceado veterinario).

Los resultados obtenidos para cada constante fisiológica fueron resumidos mediante medidas de tendencia central (media) y medidas de dispersión (desviación estándar y rango). Se determinó la diferencia entre los valores hematológicos y bioquímicos por efecto del sexo mediante la prueba de estadística de T de Student y por efecto del tamaño y tipo de alimentación, con la prueba de Análisis de Varianza.

\section{RESULTADOS}

Luego de descartar los animales que presentaron alteraciones al examen clínico se quedó con 102 ejemplares de la raza Perro sin Pelo del Perú, a los cuales se les realizó un hemograma descartándose 11 animales que presentaron alteraciones de importancia en los resultados, más de dos valores de desviación estándar del rango referencial (Davidson et al., 1998), quedando con una muestra final de 91 animales.

El promedio y rangos de los valores eritrocitarios (hemoglobina, hematocrito, número de eritrocitos, volumen globular medio y concentración de hemoglobina globular media) y leucocitarios (número de leucocitos, abastonados, segmentados, linfocitos, monocitos, eosinófilos, basófilos, y plaquetas) estuvieron dentro de rango referencial para la especie (Davidson et al., 1998), aunque se observaron algunas variaciones no estadísticas para la lectura del hemograma (ver cuadros).

El promedio y rangos de los valores bioquímicos séricos (úrea, creatinina, alanina aminotransferasa, gamma-glutamiltranspeptidasa, proteínas totales, albúmina y globulinas) mostraron incremento para los resultados úrea y ALT, y resultados por debajo del rango para creatinina y proteínas totales y fraccionadas en comparación con el referencial para la especie (Davidson et al., 1998). Los valores elevados de úrea y ALT solo se encuentran dentro del rango normal en los ejemplares que consumían alimentación mixta Se encontró diferencias para hemoglobina, eritrocitos, segmentados, úrea y globulinas en referencia a la variables sexo. También se encontró diferencias estadísticas para hematocrito, linfocitos, úrea, albúmina y globulinas cuando se contrasto con la variable tamaño; y con volumen globular medio (VGM), segmentados, monocitos, úrea y proteínas totales cuando se contrastó con la variable alimentación (ver cuadros) 
Cuadro 1. Media y desviación estándar del recuento de la biometría hemática en la raza PSPP diferenciado por sexo, tamaño y tipo de alimentación

\begin{tabular}{|c|c|c|c|c|c|c|}
\hline Biome tría Hemática & $\begin{array}{c}\text { Tamaño } \\
\text { de muestra }\end{array}$ & $\begin{array}{c}\text { Hb } \\
(\mathrm{g} / \mathrm{dl})\end{array}$ & $\begin{array}{l}\mathrm{Ht} \\
(\%)\end{array}$ & $\begin{array}{c}\text { Eritrocito } \\
\quad\left(\mathrm{ul}^{\wedge} \mathbf{6}\right)\end{array}$ & $\begin{array}{c}\text { VGM } \\
\text { (fl) }\end{array}$ & $\begin{array}{c}\text { CHGM } \\
\text { (g/dl) }\end{array}$ \\
\hline \multicolumn{7}{|l|}{ Sexo: } \\
\hline Macho & $(\mathrm{n}=45)$ & $15.8 \pm 2.5^{* *, \mathrm{a}}$ & $45.5 \pm 5.3$ & $6.6 \pm 0.9^{\mathrm{a}}$ & $63.7 \pm 5.8^{*}$ & $35.2 \pm 5.4^{* * *}$ \\
\hline He mbra & $(n=46)$ & $14.8 \pm 2.2^{\mathrm{b}}$ & $43.8 \pm 4.9$ & $6.2 \pm 0.8^{*, b}$ & $64.9 \pm 6.1^{*}$ & $34.3 \pm 4.2^{* * *}$ \\
\hline \multicolumn{7}{|l|}{ Tamaño: } \\
\hline Pequeño & $(n=29)$ & $14.6 \pm 1.7$ & $42.4 \pm 5.0^{\mathrm{b}}$ & $6.2 \pm 0.8^{*}$ & $64.0 \pm 4.3^{*}$ & $35.0 \pm 4.4^{* * *}$ \\
\hline Mediano & $(n=43)$ & $15.4 \pm 2.5$ & $45.2 \pm 5.4^{\mathrm{a}, \mathrm{b}}$ & $6.5 \pm 0.9$ & $64.7 \pm 7.1^{*}$ & $34.7 \pm 4.8^{* * *}$ \\
\hline Grande & $(\mathrm{n}=19)$ & $16.1 \pm 2.9^{* *}$ & $46.7 \pm 3.6^{\mathrm{a}}$ & $6.7 \pm 0.9$ & $63.8 \pm 5.4^{*}$ & $34.3 \pm 5.4^{* * *}$ \\
\hline \multicolumn{7}{|l|}{ Alimentación: } \\
\hline Casera & $(n=72)$ & $15.5 \pm 2.3$ & $44.2 \pm 4.9$ & $6.4 \pm 0.8$ & $63.5 \pm 4.2^{*, \mathrm{~b}}$ & $34.7 \pm 4.5^{* * *}$ \\
\hline Balance ada & $(\mathrm{n}=10)$ & $15.6 \pm 3.0$ & $45.7 \pm 4.3$ & $6.6 \pm 1.1$ & $64.8 \pm 7.7^{*, \mathrm{~b}}$ & $34.6 \pm 5.8^{* * *}$ \\
\hline Mixta & $(\mathrm{n}=9)$ & $16.1 \pm 2.6^{*}$ & $47.3 \pm 7.3$ & $6.4 \pm 1.3^{*}$ & $70.3 \pm 11.2^{* * *, a}$ & $34.9 \pm 6.1^{* * *}$ \\
\hline Promedio & $(\mathrm{n}=91)$ & $15.3 \pm 2.4$ & $44.6 \pm 5.2$ & $6.4 \pm 0.9$ & $64.3 \pm 5.9^{*}$ & $34.7 \pm 4.8^{* * *}$ \\
\hline Rango Referencial $^{1}$ & & $12-18$ & $37-55$ & $5.5-8.5$ & $60-77$ & $32-37$ \\
\hline
\end{tabular}

Hb: Hemoglobina; Ht: Hematocrito; VGM: Volumen Globular Medio; CHGM: Concentración de Hemoglobina Corpuscular Media.

${ }^{1}$ Rango Referencial para la especie canina obtenido de Davidson et al., 1998.

* El rango resultante de las muestras se encuentra por debajo del límite inferior del rango referencial para la especie.

** El rango resultante de las muestras se encuentra por encima del límite superior del rango referencial para la especie.

*** El rango resultante de las muestras es más amplio que el rango referencial para la especie.

${ }^{\mathrm{a}, \mathrm{b}}$ Letras diferentes indican que las medidas para los estratos de la variable evaluada son diferentes $(\mathrm{p}<0.05)$.

Cuadro 2. Media y desviación estándar del recuento leucocitario en la raza PSPP diferenciado por sexo, tamaño y tipo de alimentación

\begin{tabular}{|c|c|c|c|c|c|c|c|c|c|}
\hline Recuento Leucocitario & $\begin{array}{c}\text { Tamaño } \\
\text { de muestra }\end{array}$ & $\begin{array}{c}\text { Leucocitos } \\
\left(\mathbf{1 0}^{\wedge} \mathbf{3}\right)\end{array}$ & $\begin{array}{c}\text { Abastonados } \\
\text { (\%) }\end{array}$ & $\begin{array}{c}\text { Segmentados } \\
(\%)\end{array}$ & $\begin{array}{c}\text { Linfocitos } \\
(\%)\end{array}$ & $\begin{array}{c}\text { Monocitos } \\
\text { (\%) }\end{array}$ & $\begin{array}{c}\text { Eosinófilos } \\
(\%)\end{array}$ & $\begin{array}{c}\text { Basófilos } \\
(\%)\end{array}$ & $\begin{array}{c}\text { Plaquetas } \\
\quad\left(\text { ul`}^{\wedge} 6\right)\end{array}$ \\
\hline \multicolumn{10}{|l|}{ Sexo: } \\
\hline Macho & $(\mathrm{n}=45)$ & $12.6 \pm 2.4$ & $0.0 \pm 0.1$ & $77.6 \pm 9.1^{* *, \&, a}$ & $13.1 \pm 7.3^{*}$ & $4.9 \pm 3.2^{*}$ & $4.3 \pm 3.2^{*}$ & $0.0 \pm 0.2$ & $3.5 \pm 1.2$ \\
\hline Hembra & $(n=46)$ & $11.8 \pm 3.1$ & $0.0 \pm 0.2$ & $74.4 \pm 6.4^{* *, b}$ & $16.8 \pm 6.1^{*}$ & $4.3 \pm 2.5^{*}$ & $4.4 \pm 3.7^{*}$ & $0.0 \pm 0.0$ & $3.5 \pm 1.2$ \\
\hline \multicolumn{10}{|l|}{ Tamaño: } \\
\hline Pequeño & $(n=29)$ & $12.9 \pm 3.2$ & $0.0 \pm 0.2$ & $73.5 \pm 7.3^{* *}$ & $18.2 \pm 6.7^{*, \mathrm{a}}$ & $3.9 \pm 1.9^{*}$ & $4.3 \pm 3.8^{*}$ & $0.0 \pm 0.0$ & $3.5 \pm 1.4$ \\
\hline Mediano & $(n=43)$ & $12.1 \pm 2.4$ & $0.1 \pm 0.1$ & $78.3 \pm 7.9^{* *, \&}$ & $12.9 \pm 6.3^{*, b}$ & $4.7 \pm 3.0^{*}$ & $4.0 \pm 3.0^{*}$ & $0.0 \pm 0.2$ & $3.7 \pm 1.1$ \\
\hline Grande & $(\mathrm{n}=19)$ & $11.1 \pm 2.5$ & $0.0 \pm 0.0$ & $74.7 \pm 8.0^{* *}$ & $14.7 \pm 7.2^{*, b}$ & $5.4 \pm 3.6^{*}$ & $5.2 \pm 3.9^{*}$ & $0.1 \pm 0.2$ & $3.1 \pm 0.6$ \\
\hline \multicolumn{10}{|l|}{ Alimentación: } \\
\hline Casera & $(n=72)$ & $12.2 \pm 2.8$ & $0.0 \pm 0.2$ & $74.5 \pm 7.1^{* *, a, b}$ & $16.0 \pm 6.6^{*}$ & $4.8 \pm 2.8^{*, \mathrm{a}}$ & $4.6 \pm 3.6^{*}$ & $0.0 \pm 0.2$ & $3.5 \pm 1.2$ \\
\hline Balanceada & $(n=10)$ & $11.1 \pm 1.4$ & $0.0 \pm 0.0$ & $78.3 \pm 6.9^{* *, \&, b}$ & $11.7 \pm 6.0^{*}$ & $5.4 \pm 3.4^{*, \mathrm{a}}$ & $4.6 \pm 3.1^{*}$ & $0.0 \pm 0.0$ & $3.5 \pm 0.9$ \\
\hline Mixta & $(n=9)$ & $12.8 \pm 3.3$ & $0.1 \pm 0.3$ & $85.6 \pm 9.3^{* *, \&, a}$ & $10.3 \pm 8.0^{*}$ & $2.0 \pm 1.4^{*, \mathrm{~b}}$ & $2.0 \pm 1.3^{*}$ & $0.0 \pm 0.0$ & $4.0 \pm 1.2^{* *}$ \\
\hline Promedio & $(\mathrm{n}=91)$ & $12.2 \pm 2.8$ & $0.0 \pm 0.2$ & $76.0 \pm 8.0^{* * *}$ & $15.0 \pm 6.9^{*}$ & $4.6 \pm 2.9^{*}$ & $4.3 \pm 3.4^{*}$ & $0.0 \pm 0.1$ & $3.5 \pm 1.2$ \\
\hline Rango Referencial ${ }^{1}$ & & $6.0-17.0$ & $0-3$ & $60-77$ & $12-30$ & $3-10$ & $2-10$ & $0-1$ & $2.0-5.0$ \\
\hline
\end{tabular}

${ }^{1}$ Rango Referencial para la especie canina obtenido de Davidson et al., 1998.

* El rango resultante de las muestras se encuentra por debajo del límite inferior del rango referencial para la especie.

** El rango resultante de las muestras se encuentra por encima del límite superior del rango referencial para la especie.

${ }^{\&}$ La media se encuentra por encima del rango referencial para la especie.

${ }^{a, b}$ Letras diferentes indican que las medidas para los estratos de la variable evaluada son diferentes $(p<0.05)$. 
Cuadro 3. Media y desviación estándar del recuento leucocitario en valor absoluto en la raza PSPP diferenciado por sexo, tamaño y tipo de alimentación

\begin{tabular}{|c|c|c|c|c|c|c|c|c|c|}
\hline Recuento Leucocitario & $\begin{array}{c}\text { Tamaño } \\
\text { de muestra }\end{array}$ & $\begin{array}{c}\text { Leucocitos } \\
\quad\left(\mathrm{ul}^{\wedge} \mathbf{3}\right)\end{array}$ & $\begin{array}{c}\text { Abastonados } \\
\text { (ul) }\end{array}$ & $\begin{array}{c}\text { Segmentados } \\
\left(\mathrm{ul}^{\wedge} 3\right)\end{array}$ & $\begin{array}{c}\text { Linfocitos } \\
\quad\left(\mathrm{ul}^{\wedge} 3\right)\end{array}$ & $\begin{array}{c}\text { Monocitos } \\
\quad\left(\mathrm{ul}^{\wedge} 3\right)\end{array}$ & $\begin{array}{c}\text { Eosinófilos } \\
\quad\left(\mathrm{ul}^{\wedge} \mathbf{3}\right)\end{array}$ & $\begin{array}{c}\text { Basófilos } \\
\text { (ul) }\end{array}$ & $\begin{array}{c}\text { Plaquetas } \\
\quad\left(\mathrm{ul}^{\wedge} 6\right)\end{array}$ \\
\hline \multicolumn{10}{|l|}{ Sexo: } \\
\hline Macho & $(n=45)$ & $12.6 \pm 2.4$ & $1.6 \pm 11.0$ & $9.8 \pm 2.4^{* *, a}$ & $1.6 \pm 0.9^{*}$ & $0.6 \pm 0.4$ & $0.5 \pm 0.4$ & $6.3 \pm 29.8$ & $3.5 \pm 1.2$ \\
\hline Hembra & $(n=46)$ & $11.8 \pm 3.1$ & $4.9 \pm 23.6$ & $8.7 \pm 2.4^{b}$ & $1.9 \pm 0.8$ & $0.5 \pm 0.3$ & $0.5 \pm 0.5^{*}$ & $0.0 \pm 0.0$ & $3.5 \pm 1.2$ \\
\hline \multicolumn{10}{|l|}{ Tamaño: } \\
\hline Pequeño & $(\mathrm{n}=29)$ & $12.9 \pm 3.2$ & $3.3 \pm 17.6$ & $9.5 \pm 2.7^{* *}$ & $2.3 \pm 0.8^{\mathrm{a}}$ & $0.5 \pm 0.3$ & $0.6 \pm 0.6$ & $0.0 \pm 0.0$ & $3.5 \pm 1.4$ \\
\hline Mediano & $(n=43)$ & $12.1 \pm 2.4$ & $4.7 \pm 22.7$ & $9.5 \pm 2.5^{* *}$ & $1.5 \pm 0.8^{*, b}$ & $0.6 \pm 0.4$ & $0.5 \pm 0.4$ & $2.9 \pm 18.8$ & $3.7 \pm 1.1$ \\
\hline Grande & $(\mathrm{n}=19)$ & $11.1 \pm 2.5$ & $0.0 \pm 0.0$ & $8.3 \pm 2.0$ & $1.6 \pm 0.8^{*}, \mathrm{~b}$ & $0.6 \pm 0.5$ & $0.6 \pm 0.5$ & $8.4 \pm 36.7$ & $3.1 \pm 0.6$ \\
\hline \multicolumn{10}{|l|}{ Alimentación: } \\
\hline Casera & $(\mathrm{n}=72)$ & $12.2 \pm 2.8$ & $2.3 \pm 14.1$ & $9.1 \pm 2.3^{\mathrm{a}, \mathrm{b}}$ & $1.9 \pm 0.8$ & $0.6 \pm 0.4^{\mathrm{a}}$ & $0.6 \pm 0.5$ & $3.9 \pm 23.6$ & $3.5 \pm 1.2$ \\
\hline Balance ada & $(\mathrm{n}=10)$ & $11.1 \pm 1.4$ & $0.0 \pm 0.0$ & $8.7 \pm 1.6^{b}$ & $1.3 \pm 0.6^{*}$ & $0.6 \pm 0.3^{\mathrm{a}}$ & $0.5 \pm 0.4$ & $0.0 \pm 0.0$ & $3.5 \pm 0.9$ \\
\hline Mixta & $(\mathrm{n}=9)$ & $12.8 \pm 3.3$ & $14.6 \pm 43.7$ & $11.1 \pm 3.6^{*, \mathrm{a}}$ & $1.2 \pm 1.0^{*}$ & $0.2 \pm 0.2^{*, \mathrm{~b}}$ & $0.3 \pm 0.2$ & $0.0 \pm 0.0$ & $4.0 \pm 1.2^{* *}$ \\
\hline Promedio & $(\mathrm{n}=91)$ & $12.2 \pm 2.8$ & $3.3 \pm 18.5$ & $9.3 \pm 2.5$ & $1.8 \pm 0.8$ & $0.6 \pm 0.4$ & $0.5 \pm 0.5^{*}$ & $3.1 \pm 21.0$ & $3.5 \pm 1.2$ \\
\hline Rango Referencial $^{1}$ & & $6.0-17.0$ & $0-300$ & $3.0-11.5$ & $1.0-4.8$ & $0.2-1.5$ & $0.1-1.3$ & $0-100$ & $2.0-5.0$ \\
\hline
\end{tabular}

${ }^{1}$ Rango Referencial para la especie canina obtenido de Davidson et al., 1998.

* El rango resultante de las muestras se encuentra por debajo del límite inferior del rango referencial para la especie.

** El rango resultante de las muestras se encuentra por encima del límite superior del rango referencial para la especie.

${ }^{a, b}$ Letras diferentes indican que las medidas para los estratos de la variable evaluada son diferentes $(p<0.05)$.

Cuadro 4. Media y desviación estándar de la bioquímica sérica (perfil hepático y renal) en la raza PSPP diferenciado por sexo, tamaño y tipo de alimentación

\begin{tabular}{|c|c|c|c|c|c|c|c|c|}
\hline Bioquímica Sérica & $\begin{array}{c}\text { Tamaño } \\
\text { de muestra }\end{array}$ & $\begin{array}{c}\text { Urea } \\
(\mathrm{mg} / \mathrm{dl})\end{array}$ & $\begin{array}{c}\text { Cre atinina } \\
\text { (mg/dl) }\end{array}$ & $\begin{array}{l}\mathbf{A L T} \\
(\mathbf{U} / \mathbf{L})\end{array}$ & $\begin{array}{l}\text { GGT } \\
(\mathbf{U} / \mathbf{L})\end{array}$ & $\begin{array}{c}\text { Prot. Totales } \\
\text { (g/dl) }\end{array}$ & $\begin{array}{l}\text { Albúmina } \\
\text { (g/dl) }\end{array}$ & $\begin{array}{c}\text { Globulinas } \\
\text { (g/dl) }\end{array}$ \\
\hline \multicolumn{9}{|l|}{ Sexo: } \\
\hline Macho & $(\mathrm{n}=45)$ & $48.1 \pm 19.4^{\&, * *, a}$ & $0.3 \pm 0.5^{*}$ & $56.3 \pm 23.0^{* *}$ & $4.2 \pm 3.0$ & $5.7 \pm 0.7^{*}$ & $2.8 \pm 0.5^{*}$ & $2.5 \pm 0.9^{*, a}$ \\
\hline Hembra & $(n=46)$ & $37.9 \pm 14.6^{* *, b}$ & $0.2 \pm 0.4^{*}$ & $54.3 \pm 28.3^{* * *}$ & $3.7 \pm 3.9$ & $5.6 \pm 0.6^{*}$ & $3.0 \pm 0.6^{*}$ & $2.1 \pm 0.7^{*, b}$ \\
\hline \multicolumn{9}{|l|}{ Tamaño: } \\
\hline Pequeño & $(\mathrm{n}=29)$ & $44.1 \pm 14.5^{\&, * *}$ & $0.1 \pm 0.3^{*}$ & $62.9 \pm 30.1^{* * *}$ & $5.0 \pm 4.5$ & $5.7 \pm 0.6^{*}$ & $3.1 \pm 0.5^{*, \mathrm{a}}$ & $2.0 \pm 0.7^{*, b}$ \\
\hline Mediano & $(n=43)$ & $42.6 \pm 20.8^{\&, * *}$ & $0.3 \pm 0.4^{*}$ & $51.9 \pm 23.6^{* *}$ & $3.6 \pm 2.9$ & $5.7 \pm 0.7^{*}$ & $2.8 \pm 0.5^{*, a, b}$ & $2.5 \pm 0.8^{*, a}$ \\
\hline Grande & $(n=19)$ & $42.0 \pm 15.7^{\&, * *}$ & $0.3 \pm 0.5^{*}$ & $51.3 \pm 21.4^{* * *}$ & $3.2 \pm 2.2$ & $5.7 \pm 0.7^{*}$ & $2.6 \pm 0.8^{*, b}$ & $2.5 \pm 0.8^{*}, \mathrm{a}$ \\
\hline \multicolumn{9}{|l|}{ Alimentación: } \\
\hline Casera & $(\mathrm{n}=72)$ & $45.0 \pm 17.9^{\&, * *, a}$ & $0.2 \pm 0.4^{*}$ & $57.0 \pm 27.1^{* * *}$ & $4.1 \pm 3.5$ & $5.6 \pm 0.7^{*, a, b}$ & $2.9 \pm 0.6^{*}$ & $2.3 \pm 0.8^{*}$ \\
\hline Balance ada & $(\mathrm{n}=10)$ & $42.8 \pm 17.7^{\&, * *, a, b}$ & $0.4 \pm 0.5^{*}$ & $54.4 \pm 24.4^{* *}$ & $3.3 \pm 2.8$ & $6.1 \pm 0.6^{*, \mathrm{a}}$ & $2.8 \pm 0.6^{*}$ & $2.8 \pm 0.6^{*}$ \\
\hline Mixta & $(\mathrm{n}=9)$ & $27.1 \pm 7.6^{b}$ & $0.3 \pm 0.5^{*}$ & $42.4 \pm 6.8$ & $3.2 \pm 3.6$ & $5.4 \pm 0.5^{*, b}$ & $3.0 \pm 0.5^{*}$ & $2.2 \pm 0.8^{*}$ \\
\hline Promedio & $(\mathrm{n}=91)$ & $43.0 \pm 17.8^{\&, * *}$ & $0.2 \pm 0.4^{*}$ & $55.3 \pm 25.7^{* *}$ & $3.9 \pm 3.4$ & $5.7 \pm 0.7^{*}$ & $2.9 \pm 0.6^{*}$ & $2.3 \pm 0.8^{*}$ \\
\hline Rango Referencial $^{1}$ & & $20-40$ & $0.5-1.6$ & $19-70$ & $0-10$ & $5.4-7.4$ & $2.9-4.2$ & $2.3-4.4$ \\
\hline
\end{tabular}

ALT: Alanino Aminotransferasa; GGT: Gamm-0glutamil Transpeptidas; Prot. Totales: Proteínas Totales.

${ }^{1}$ Rango Referencial para la especie canina obtenido de Davidson et al., 1998.

* El rango resultante de las muestras se encuentra por debajo del límite inferior del rango referencial para la especie.

** El rango resultante de las muestras se encuentra por encima del límite superior del rango referencial para la especie.

${ }^{\&}$ La media se encuentra por encima del rango referencial para la especie.

${ }^{\mathrm{a}, \mathrm{b}}$ Letras diferentes indican que las medidas para los estratos de la variable evaluada son diferentes $(\mathrm{p}<0.05)$. 


\section{DISCUSIÓN}

Si bien el análisis estadístico muestra diferencias estadísticas para el efecto del sexo sobre la concentración de hemoglobina y número de eritrocitos ninguna de ellas escapa al rango normal de la especie (Davidson et al. 1998), por lo que estos resultados no poseen significancia biológica; así mismo, estudios realizados por Ariyibi et al. (2002), Khan et al. (2011) y Olayemi e Ighagbon (2011) corroboran estos resultados. Igualmente, el análisis de varianza reveló diferencia estadística para los niveles de hematocrito en referencia al tamaño; sin embargo, tampoco posee significancia biológica por encontrarse dentro del rango referencial para la especie (Davidson et al., 1998). Así mismo, Ariyibi et al. (2002) y Olayemi e Ighagbon (2011) no encontraron diferencias entre razas corroborando esta afirmación.

Asi mismo, se encontró diferencias estadísticas para el VGM, neutrófilos segmentados y monocitos en referencia a la alimentación siendo significativas para los dos últimos, esta no debería ser concluyente como característica de la raza dado que el resto de valores de la biometría hemática se encuentran dentro de los rangos referenciales para la especie (Davidson et al., 1998) además debemos considerar que en este estudio la alimentación no fue una variable controlada.

El VGM se puede ver afectado por diversas condiciones, como son: la deficiencia de hierro (Davidson et al., 1998), el sistema de contaje de células hematológicas automatizadas en comparación con la fórmula tradicional (Willard et al., 1999), en razas como en Akita Japonés y Shibas que presentan valor menor al referencial para la especie (Davidson et al., 1998); y el American Pitbull Terrier que posee un mayor intervalo en referencia a razas como Lobero Irlandés, Beagle y mixtas; sin embargo, no fueron diferente al de Coyotes y la raza Greyhound (Aengwanich et al., 2007), y así mismo en condiciones como preñez y estado corporal en el Pastor Alemán (Khan et al., 2011). En este estudio los resultados obtenidos hacen probable que la raza presente una mínima variación en el tamaño de sus eritrocitos, que no fue detectable por el sistema de conteo en la lectura utilizado en este trabajo; aunque se requeriría comparar con el análisis de biometría hemática automatizada.

Por otro lado, Davidson et al. (1998) atribuye la variación en la variable tipo de alimentación para los neutrófilos segmentados y monocitos a factores como el estrés, siendo probable que el tiempo en que se tomó las muestras de sangre algunas mascotas se hayan encontrado bajo estas condiciones, debido a su temperamento nervioso de la raza.

En relación a los neutrófilos segmentados también se encontró con variaciones estadísticas en relación a la variable sexo, para la prueba de $\mathrm{T}$ de Student, siendo mayor para los machos, en concordancia con Aengwanich et al. (2007), quién encontró un mayor número de células blancas en American Pitbull machos, en comparación con hembras.

Así mismo, se encontró una variación estadística de los linfocitos en relación al tamaño, siendo estos de mayor tamaño en los especímenes de talla pequeña, pero no se han encontrado reportes que expliquen este hallazgo.

La prueba de $\mathrm{T}$ de Student también reveló un diferencia estadística para úrea en la variable sexo, siendo mayor en los machos. Existen pocos reportes previos donde la variación de los niveles de la úrea se ven afectados por el sexo; no obstante, en un estudio comparativo entre la raza Pastor Alemán y perros de la ciudad de Ibadan, Nigeria, no mostró diferencia estadística entre ambas razas, aunque dentro de la raza Pastor Alemán se encontró niveles más altos de úrea en los machos en comparación con las hembras (Ariyibi et al., 2002).

Del mismo modo, los niveles de úrea en sangre mostraron diferencia influenciada por la variable tamaño; sin embargo, no hay información previa acerca de este efecto. La variable tipo de alimentación para los grupos de comida casera y balanceada también se vio levemente incrementada, siendo incluso mayor para los que se alimentaban con comida casera. Un exceso de consumo de proteínas en la dieta origina un incremento de úrea excretada por el riñón (Davidson et al., 1998).

De otro lado Willard et al. (1999), mencionan como una ventaja de la evaluación de la úrea cuando esta es usada como control de la dieta proteica en una mascota. En cuanto a actividad física, las carreras prolongadas han sido relacionadas con la disminución de la úrea. Asimismo, los rangos de producción y excreción de úrea en el cuerpo no son constantes durante el día, y luego de una comida con mucha proteína, la máxima concentración de úrea es obtenida luego de 8 horas (Davidson et al., 1998), en el presente estudio la toma de muestras fue en horas variadas por lo que este factor puede ser motivo de la variación encontrada en los resultados de este análisis. 
Sí bien se encontró también una variación significativa para las globulinas en relación a la variable sexo siendo superior en los machos, esta no escapa del rango referencial para la especie (Davidson et al., 1998), por lo que no posee significancia biológica; sin embargo, solo se ha reportado niveles más altos de proteínas y albúmina en hembras en comparación con machos (Ariyibi et al., 2002).

Para la variable tamaño también se ven afectados significativamente la albúmina y las globulinas, mostrando un proporción opuesta con respecto a los tamaños. No se ha encontrado referencia bibliográfica para contrastar estos resultados. La medición de ambas se encuentra más relacionada con el tipo de alimentación y edad del individuado en evaluación. Así mismo, no podemos concluir que sea característico de la raza debido a que las proteínas totales para esta variable se encuentran dentro del rango referencial (Davidson et al., 1998).

Se concluye que los valores encontrados en el presente estudio pueden ser considerados como referencia para la raza PSPP. La úrea, recuento de leucocitos y proteínas totales y fraccionadas presentaron valores fuera del rango normal para la especie, requiriéndose realizar estudios complementarios con variables controladas, para determinar si es una característica propia de la raza. El VGM y la CHGM presentaron valores fuera del rango referencial para la especie, requiriéndose realizar estudios complementarios que utilicen sistemas de conteo más sensibles y donde el procesamiento de la muestra sea inmediato.

\section{Correspondencia:}

Giannina Cortés

gcorteschinchayan@gmail.com

\section{REFERENCIAS BIBLIOGRÁFICAS}

1. Aengwanich W, Daungduen C, Pamok S, Suppaso D. 2007. Blood cell characteristics and some hematological values of American Pit-bull Terriers in Thailand. World Applied Sciences Journal 2(3): 158162.

2. Ariyibi A, Oyeyemi MO, Ajadi RA. 2002. A comparative study of some hematology and biochemical parameters of clinically healthy Alsatian and local dogs. African Journal of Biomed Research 5: 145-147.

3. Benjamín M. 1984. Manual de Patología Clínica en Veterinaria.Editorial Limusa.
4. Coppo JA, Mussart NB. 2000. Apoyatura bioquímica al diagnóstico veterinario. Casuística registrada tras 25 años de funcionamiento de un servicio de análisis clínicos. Rev. Vet. 10: 34-39.

5. Davidson M, Else R, Lumsden J. 1998. Manual of Small Animal Clinical Pathology. British Small Animal Veterinary Association.

6. Gálvez C. 2006. Manual de crianza del Perro sin Pelo del Perú. Perú: Capac Cuna. p 92.

7. Giannini EG, Testa R, Savarino V. 2005. Liver enzyme alteration: A guide for clinicians. Canadian medical association journal 172(3): 367-379.

8. Harvey J, Meyer D. 2000. El Laboratorio en Medicina Veterinaria. Interpretación y Diagnóstico. Segunda Edición. Argentina: Inter-Médica.

9. Kaneko J, Harvey J, Bruss M. 1997. Clinical Biochemistry of Domestic Animals. Academic Press.

10. Khan S, Epstein J, Olival K, Hassam M, Hossain MB, Rahman K, Elahi M, Manmun M, Haider N, Yasin G, Desmond J. 2011. Hematology and serum chemistry reference values of stray dogs in Bangldesh. Open Veterinary Journal Vol 1: 13- 20.

11. Kimura T., Ohshima S., Doi K. 1993. The inheritance and breeding results of hairless descendants of Mexican hairless dogs. Lab Anim. 27: 55-58.

12. Maniero E. 2004. Introducción a la morfología. Perú: Universidad Alas Peruanas. p 199-232.

13. Mecklenburg L. 2006. An overview on congenital alopecia in domestic animals. Veterinary dermatology 17(6): 393-410.

14. Navarro V., Popescu B., Zurita A. 1999. El papel de los tests complementarios en la modificación del diagnóstico inicial. Actas de Ciencia \& Técnica UNNE 3: 33-35.

15. Omer S. 2009. Normal Values of some serochemical parameters in male and female German Shepherd dogs in Sudan. Assiut Veterinary Medical Journal 55.120: 110-115.

16. Olayemi F, Ighagbon F. 2011. Haematology of the German Shepherd dog in a humid tropical environment. Comparative clinical pathology 20.1: 61-64.

17. Sodikoff C. 2001.Pruebas Diagnósticas y de Laboratorio en Pequeños Animales. Elsevier.

18. Steel R, Torrie J, Martínez R. 1992. Bioestadística: principios y procedimientos. McGraw-Hill.

19. Weiss P. 1970. El Perro Peruano sin Pelo (perro chino, viringo, ccala o ccalato). Revista Acta Herediana 3:33-54.

20. Willard M, Tvedten H, Turnwald G. 1999.Small Animal Clinical Diagnosis by Laboratory Methods. Saunders.

Recibido 22/07/2014

Aceptado 09/02/2015 